\title{
Comparison of Root Images between Post-Myelographic Computed Tomography and Magnetic Resonance Imag- ing in Patients with Lumbar Radiculopathy
}

\author{
Chun-Kun Park, M.D., Ph.D., ${ }^{1}$ Hong-Jae Lee, M.D., Ph.D., ${ }^{2}$ Kyeong-Sik Ryu, M.D., Ph.D. ${ }^{3}$ \\ Good Doctor Teun Teun Hospital,' Anyang, Korea \\ Department of Neurosurgery, ${ }^{2}$ Daejeon St. Mary's Hospital, College of Medicine, The Catholic University of Korea, Daejeon, Korea \\ Department of Neurosurgery, ${ }^{3}$ Seoul St. Mary's Hospital, College of Medicine, The Catholic University of Korea, Seoul, Korea
}

Objective : To evaluate the diagnostic value of computed tomography-myelography (CTM) compared to that of magnetic resonance imaging (MRI) in patients with lumbar radiculopathy.

Methods : The study included 91 patients presenting with radicular leg pain caused by herniated nucleus pulposus or lateral recess stenosis in the lumbar spine. The degree of nerve root compression on MRI and CTM was classified into four grades. The results of each imaging modality as assessed by two different observers were compared. Visual analog scale score for pain and electromyography result were the clinical parameters used to evaluate the relationships between clinical features and nerve root compression grades on both MRI and CTM. These relationships were quantified by calculating the receiver-operating characteristic curves, and the degree of relationship was compared between MRI and CTM.

Results : McNemar's test revealed that the two diagnostic modalities did not show diagnostic concurrence $(p<0.0001)$. Electromyography results did not correlate with grades on either MRI or CTM. The visual analog pain scale score results were correlated better with changes of the grades on CTM than those on MRI $(p=0.0007)$.

Conclusion : The present study demonstrates that CTM could better define the pathology of degenerative lumbar spine diseases with radiculopathy than MRI. CTM can be considered as a useful confirmative diagnostic tool when the exact cause of radicular pain in a patient with lumbar radiculopathy cannot be identified by using MRI. However, the invasiveness and potential complications of CTM are still considered to be pending questions to settle.

Key Words : Radiculopathy · Myelography $\cdot$ Magnetic resonance imaging.

- Received : September 26, 2016 •Revised : January 23, 2017 •Accepted : February 21, 2017

- Address for reprints : Hong-Jae Lee, M.D., Ph.D.

Department of Neurosurgery, Daejeon St. Mary's Hospital, College of Medicine, The Catholic University of Korea, 64 Daeheung-ro, Jung-gu, Daejeon 34943, Korea Tel : +82-42-220-9525, Fax: +82-42-222-6601, E-mail : kosailee73@gmail.com

This is an Open Access article distributed under the terms of the Creative Commons Attribution Non-Commercial License (http://creativecommons.org/licenses/by-nc/4.0) which permits unrestricted non-commercial use, distribution, and reproduction in any medium, provided the original work is properly cited. 


\section{INTRODUCTION}

Radicular pain in the leg, a representative symptom of degenerative lumbar spine diseases, results from nerve root compression or irritation at various locations by diverse pathologies. An accurate understanding of the pathology compromising the nerve root is essential to obtain good surgical outcomes ${ }^{5)}$.

Magnetic resonance imaging (MRI) is commonly used for diagnostic imaging of radicular pain in the leg because of its noninvasiveness, no radiation exposure, and superiority of soft tissue analysis. MRI is also recognized as the most accurate imaging study ${ }^{7,8,15)}$. Computed tomography-myelography (CTM), another common imaging modality, can provide good quality images of bone or calcified lesions, and deliver an image with high spatial and contrast resolution in thinner imaging slices even when metallic implants are present ${ }^{10,23)}$. Currently, its use has been limited because it entails potential complications related to lumbar puncture, intrathecal contrast injection, and radiation exposure; it is considerably more invasive and risky than MRI; and it is recognized as a less accurate imaging study than MRI.

MRI is routinely used in final decision making for surgical treatment of degenerative spinal diseases. However, some clinicians believe that CTM is more valuable than MRI for differential diagnosis between pure and calcified/ossified disc materials, to confirm bony changes in various lumbar degenerative diseases and in the diagnosis of the main pathology of radicular pain in a leg ${ }^{23)}$. From a surgical standpoint, it is important to know which method is more accurate and efficient in confirming the pathology. The authors considered the possibility that the clinical significance of CTM has been underemphasized and retrospectively evaluated the diagnostic value of CTM in comparison with that of MRI in patients with the main symptom of clinically significant radiculopathy that required surgical management because of conservative treatment failure.

\section{MATERIALS AND METHODS}

The study included 91 consecutive patients presenting with intractable unilateral radicular leg pain caused by degenerative lumbar spinal disease who fulfilled the inclusion criteria between February 2012 and January 2013. The study included only patients with herniated nucleus pulposus $(n=73)$ and lateral recess stenosis $(n=18)$. All the patients were evaluated by using preoperative electromyography (EMG) study, visual analog scale (VAS) score for pain, MRI, and CTM, and nerve root compromise was confirmed in the operative field. During the above-mentioned period, CTM was routinely used for patients who were scheduled to undergo surgery for degenerative lumbar spinal disease in the authors' institute. This study was approved by the institutional review board of the authors' institute, and because of the retrospective nature of the study, the need for informed consent was waived.

Patients with central canal stenosis presenting with claudication as the major symptom and patients with intervertebral foraminal stenosis were excluded. Generally, intervertebral foraminal stenosis is diagnosed based on a loss of fat signal on T1 sagittal images rather than on axial images ${ }^{3,24)}$. As a comparison between grading sagittal and axial images cannot be given statistical significance and the degree of root compression on axial images was classified in the present study, cases with intervertebral foraminal stenosis were excluded. Patients with central canal stenosis were also excluded because the main symptom in central canal stenosis is claudication, and a specific nerve root cannot represent a major causative struc-

Table 1. Demographic data of the patients according to diagnosis, surgical methods, and levels

\begin{tabular}{|c|c|c|c|c|c|c|c|c|c|c|}
\hline \multirow{2}{*}{ Sex } & \multirow{2}{*}{ Age } & \multicolumn{2}{|c|}{ Preoperative Dx } & \multicolumn{3}{|c|}{ Operation title } & \multicolumn{4}{|c|}{ Pathologic level } \\
\hline & & HNP & LRS & MD & ED & MF & L2-L3 & L3-L4 & L4-L5 & L5-S1 \\
\hline Male $(n=37)$ & $51.2 \pm 17.2$ & $30(81.1)$ & $7(18.9)$ & $18(48.7)$ & $12(32.4)$ & $7(18.9)$ & $0(0.0)$ & $2(5.4)$ & $23(62.2)$ & $12(32.4)$ \\
\hline Female $(n=54)$ & $58.3 \pm 13.0$ & $43(79.6)$ & $11(20.4)$ & $30(55.6)$ & $13(24.1)$ & $11(20.3)$ & $1(1.8)$ & $6(11.1)$ & $30(55.6)$ & $17(31.5)$ \\
\hline Total $(n=91)$ & $55.4 \pm 15.2$ & $73(80.2)$ & 18 (19.8) & $48(52.7)$ & $25(27.5)$ & $18(19.8)$ & $1(1.1)$ & $8(8.8)$ & $53(58.2)$ & $29(31.9)$ \\
\hline
\end{tabular}

Values are presented as mean \pm standard deviation or number (\%). Dx : diagnosis, HNP : herniated nucleus pulposus, LRS : lateral recess stenosis, MD : microdiscectomy, ED : endoscopic discectomy, MF : microfenestration 
ture for claudication. Infection cases, revision cases, and cases with an epidural injection history were also excluded, as these can cause changes in imaging other than degenerative changes. The demographic data of the patients are listed in Table 1.

The root images on the preoperative axial MRI and CTM scans of the symptomatic side at the pathological level were classified into four grades according to the severity of nerve root compression by modifying a previous grading system as follows : grade 0, no nerve root compression (root image is well visualized); grade I, nerve root is abutted or contacted; grade II, nerve root is displaced or deformed; and grade III, definite root compression or completely nonvisualized (flattened or obliterated) root image ${ }^{1,9,20)}$ (Figs. 1-3, Table 2).

Two experienced neurosurgeons evaluated the results of all the imaging studies while blinded to the patients' clinical information. One of the surgeons performed a retest of the images after 2 weeks. Any bias that might have occurred because of the memory effect should have been minimized by the delay of 2 weeks between the two readings. The results of each imaging modality by two different observers were compared, and the interobserver and intraobserver variances were calculated.

Preoperative VAS scores and EMG results were analyzed to compare their relationships with the nerve root compression grades measured on MRI and CTM. For this purpose, the receiver-operating characteristic (ROC) curve was used. Corre-
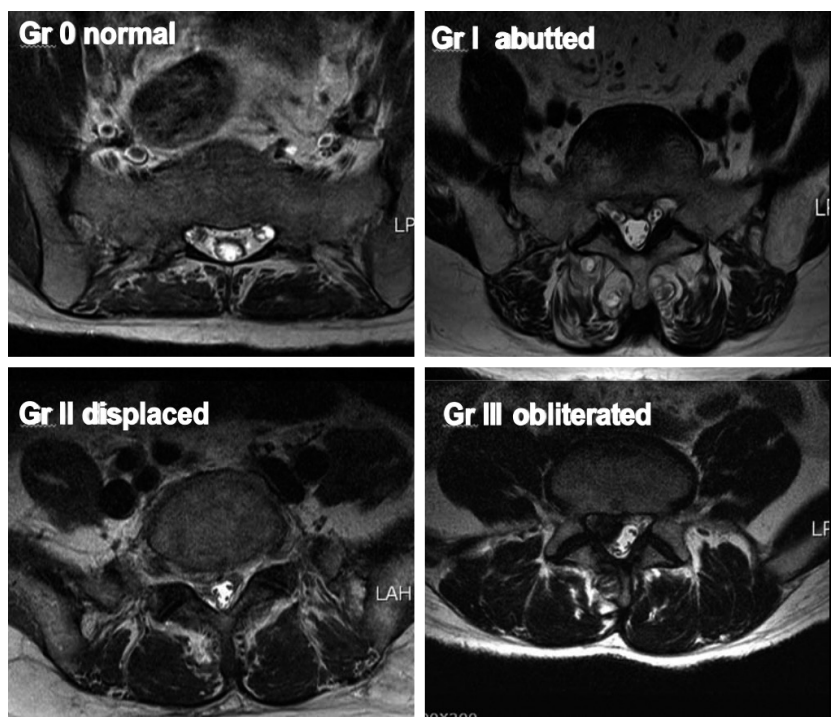

Fig. 1. Grading of nerve root images on magnetic resonance imaging. Gr : grade. lation analyses between root compression grades on MRI and clinical features were performed retrospectively by using a ROC curve. VAS score and EMG result were used as clinical parameters. In the same way, Correlation analyses between root compression grades on CTM and clinical features (VAS score and EMG result) were performed.

For the correlation analyses using ROC curves, EMG results that were congruent with clinical features were set at 1 , and the results that did not match the clinical features were set at 0 . EMG (Medelec Synergy; Cardinal Health, Surrey, UK) results were recorded and clinically assessed by a neurophysiologist. When abnormal activity on EMG at rest (fibrillation, positive sharp waves, or high-frequency repetitive discharge) or neurogenic patterns at maximum effort (fewer motor unit action potentials at high levels) were observed, radiculopathy was defined $^{6,19)}$.

VAS scores of $<6$ were set at 0 , and VAS scores of $>5$ were set at 1 . The authors set a VAS score of $\geq 6$ as the diagnostic criterion for radiculopathy, and the division was made to achieve the optimal diagnostic value (area under the curve [AUC] 0.691 , sensitivity 77.6 , specificity 62.5 ) in the ROC analysis ${ }^{11,17)}$.

We identified the complication rate and details of the complications by performing a chart review. Patient age, sex, diagnosis, surgical treatment, the cause of root compression, comorbidities, and procedure-related complications were recorded. For MRI, 3-Tesla systems (Magnetom Verio 3T; Sie-
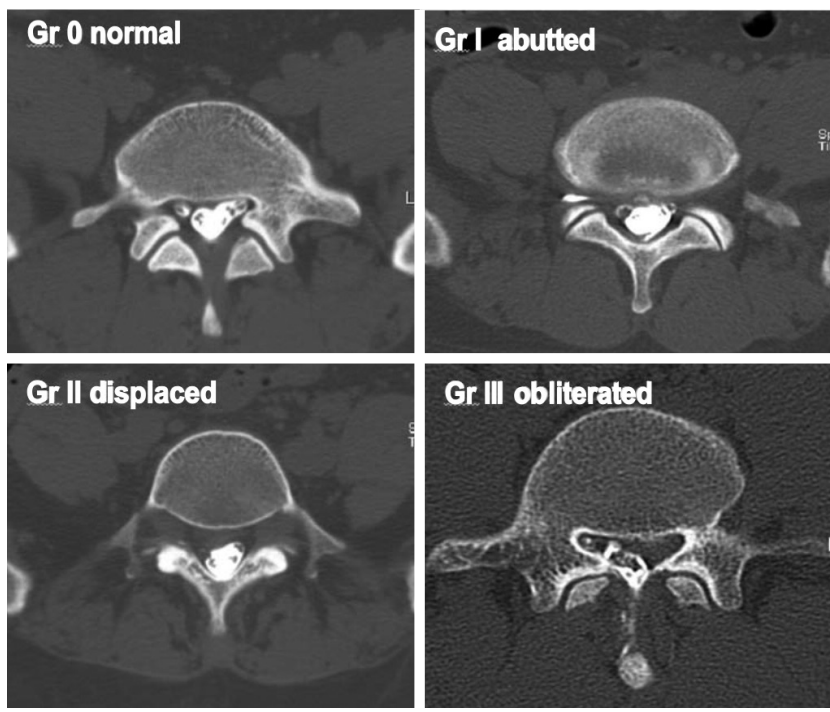

Fig. 2. Grading of nerve root images on computed tomographymyelography. Gr: grade. 
(A)

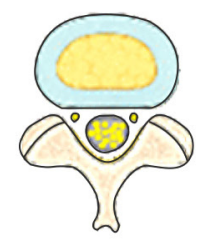

(C)

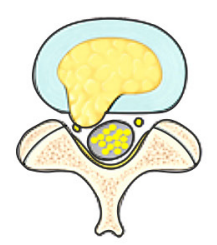

(E)

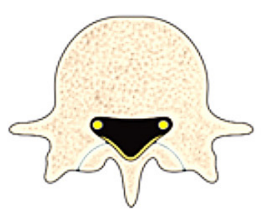

(G)

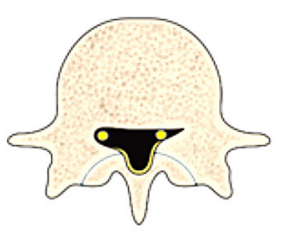

(1)

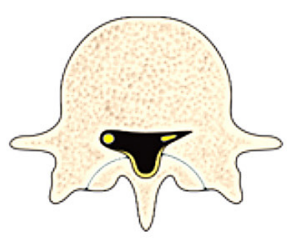

(K)

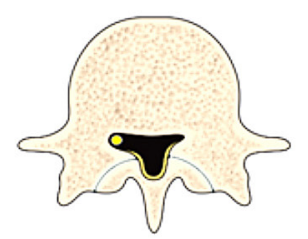

(B)

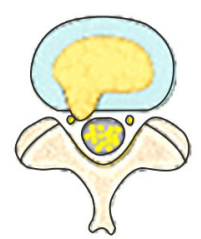

(D)

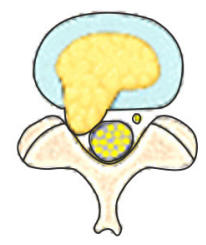

(F)

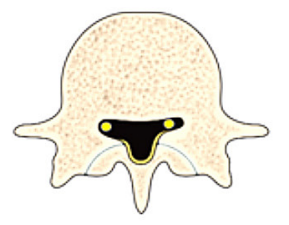

(H)

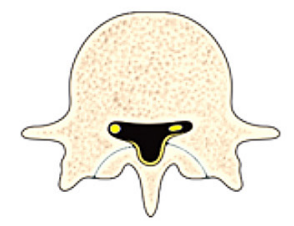

(J)

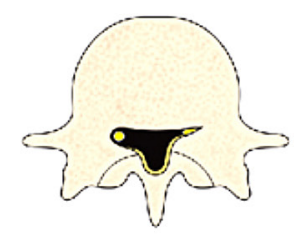

Fig. 3. Grading of nerve root images in the herniated nucleus pulposus and lateral recess stenosis. A : No compromise of the nerve root (grade 0 ). $B$ : The nerve root is abutted but does not show any signs of deviation or deformation (grade I). C : The nerve root is displaced (deviated) and deformed by compression (grade II). D : Definite nerve root compression with the nerve root completely nonvisualized (grade III). E : No compromise of the nerve root in the lateral recess (grade 0 ). F : Trefoilshape change of the lateral recess (grade I). G : Early acute angular narrowing of the lateral recess (grade I). $\mathrm{H}$ : Trefoil-shape narrowing of the lateral recess, and displaced (deviated) and deformed nerve root (grade II). I : Angular pinch-like narrowing of the lateral recess, and displaced (deviated) and deformed nerve root (grade II). J : Severe angular pinch-like narrowing of the lateral recess and flattened nerve root (grade III). $\mathrm{K}$ : The root image is completely nonvisualized in the lateral recess (grade III).

Table 2. Grading for root compression in lumbar degenerative disease: graded based on the MRI and CTM findings

\begin{tabular}{cc}
\hline Grade $\mathbf{0}$ & $\begin{array}{c}\text { - Normal imaging findings without any nerve root } \\
\text { compression and good visualization of the nerve root } \\
\text { image }\end{array}$ \\
Grade I & - The nerve root is abutted or contacted but does not \\
& show any signs of deviation or deformation \\
& - The nerve root is abutted or contacted by an herniated \\
& disc fragment \\
& - Early acute trefoil or angular shape change of the lateral \\
& recess \\
Grade II & - The nerve root is displaced (deviated) or deformed by an \\
& herniated disc fragment \\
& - The nerve root is displaced (deviated) or deformed by a \\
trefoil shape or angular pinch-like change of the lateral \\
recess
\end{tabular}

MRI : magnetic resonance imaging, CTM : computed tomographymyelography

mens, Berlin, Germany) were used; for CT, Somatom Definition AS (Siemens, Berlin, Germany) was used. T2-weighted axial MRI scans (TR/TE/NEX/FA, 4700/71 ms/2.0/160 ${ }^{\circ}$ obtained by using a $200 \times 200-\mathrm{mm}$ field of view and $4-\mathrm{mm}$ slice thickness were used for this analysis.

The myelography procedure was performed by 2 neurosurgeons with a 22- to 25-gauge styletted spinal needle using 12 $\mathrm{mL}$ of nonionic water-soluble contrast medium (Visipaque; GE Healthcare, Cork, Ireland) at the L4-L5 level under fluoroscopic guidance. CT imaging was performed as soon as possible after the myelography procedure.

Statistical verification was determined using PASW Statistics version 18.0 (SPSS Inc., Chicago, IL, USA) and MedCalc version 12.0 (MedCalc, Mariakerke, Belgium). A $p$-value of $<0.05$ was considered statistically significant. The chi-square test and t-test were used to analyze the general characteristics of the patient groups. McNemar's test was utilized to check for an association between CTM and MRI grades. Procedure-related complications were also recorded.

\section{RESULTS}

Grading of the nerve root compression on the MRI axial scans showed that among the cases, $0 \%$ was grade $0,30.8 \%$ 
were grade I, 38.5\% were grade II, and 30.8\% were grade III. On the other hand, grading of the nerve root compression on the CTM axial scans showed that among the cases, $0 \%$ was grade $0,2.2 \%$ were grade I, $30.8 \%$ were grade II, and $67 \%$ were grade III. The mean intraclass coefficients of both intrarater and interrater reliability were statistically significant (Table 3).

In $54 \%$ of the cases, two different grading results were found in the same patient, one from MRI and the other from CTM (Table 4). The diagnostic concurrence between CTM and MRI grades was confirmed by using McNemar's test, which revealed that the two diagnostic modalities did not show diagnostic concurrence of the study results $(p<0.0001)$. CTM tended to identify higher grades of nerve root compression than did MRI.

The ROC curves for analyses between MRI/CTM grades and EMG results revealed that EMG results did not correlate with changes in MRI or CTM grades (Fig. 4). The ROC curves for analyses between MRI/CTM grades and pain severity based on VAS score revealed that VAS severity was more highly correlated with changes of the grades on CTM than with those on MRI ( $p=0.0007$; Fig. 5).

From the perspective of diagnostic value, the above-mentioned results show that CTM is almost moderately accurate

Table 3. Mean ICC values for intrarater and interrater reliability with MRI and CTM

\begin{tabular}{llc}
\hline & & Mean ICC \\
\hline Intrarater reliability & CTM & $0.911(0.866-0.942)(p<0.0001)$ \\
& MRI & $0.991(0.986-0.994)(p<0.0001)$ \\
Interrater reliability & CTM & $0.924(0.884-0.950)(p<0.0001)$ \\
& MRI & $0.978(0.967-0.986)(p<0.0001)$ \\
\hline
\end{tabular}

ICC : intraclass correlation coefficient, MRI : magnetic resonance imaging, CTM : computed tomography-myelography
(AUC 0.69; 0.5<AUC<0.7), whereas MRI was less accurate (AUC 0.598; 0.5<AUC<0.7) according to a guideline by Swets ${ }^{11)}$.

Seventeen cases of procedure-related complications of CTM, including 1 case of intracranial hypotension (5\%), 14 cases of temporary back pain (82\%), 2 cases of temporary neurological change (11\%), 1 case of worsening, and 1 case of improvement of existing radicular leg pain, were found.

\section{DISCUSSION}

Accurate diagnosis of nerve root compressive pathology is crucial for operative success. Therefore, preoperative evaluation should be precise, and the results must be assessed in detail $^{5)}$. Among the imaging studies, MRI and CT are most commonly used, and MRI is currently the primary imaging modality ${ }^{7,8,15)}$. However, CTM is no longer a routine primary examination because of its invasiveness, attributable risk of radiation exposure, and potential procedure-related complications, although some spine surgeons are still confident of its diagnostic accuracy in patients with radicular leg pain ${ }^{2,13,14,18)}$.

The lateral lumbar spinal canal includes the nerve root canal (lateral recess) and the intervertebral foramen (neural foramen) $)^{3)}$. The lateral lumbar spinal canal was divided into three anatomic zones by Botwin and Gruber ${ }^{3)}$, namely the entrance zone, midzone, and exit zone. The entrance zone is the subarticular area and is synonymous with the lateral recess area $^{3)}$. In this study, cases of intervertebral foraminal stenosis were excluded because these are diagnosed based on a loss of fat signal on T1 sagittal images rather than on axial images ${ }^{3,24)}$.

Radicular leg pain, a major symptom of degenerative lumbar spinal disease, is generally caused by lumbar nerve root compression. Various pathologies cause radicular pain, including herniated discs and spinal stenosis. Radicular pain is

Table 4. Comparison of root compression grade between MRI and CTM

\begin{tabular}{|c|c|c|c|c|}
\hline \multirow{2}{*}{ СTM } & \multicolumn{4}{|c|}{ MRI } \\
\hline & Grade 0 & Grade I & Grade II & Grade III \\
\hline Grade 0 & $0(0.0)$ & $0(0.0)$ & $0(0.0)$ & $0(0.0)$ \\
\hline Grade I & $0(0.0)$ & $1(1.1)$ & $0(0.0)$ & $1(1.1)$ \\
\hline Grade II & $0(0.0)$ & 15 (16.5) & $13(14.3)$ & $0(0.0)$ \\
\hline Grade III & $0(0.0)$ & $12(13.2)$ & $22(24.2)$ & $27(29.7)$ \\
\hline
\end{tabular}

Values are presented as number (\%). MRI : magnetic resonance imaging, CTM : computed tomography-myelography 

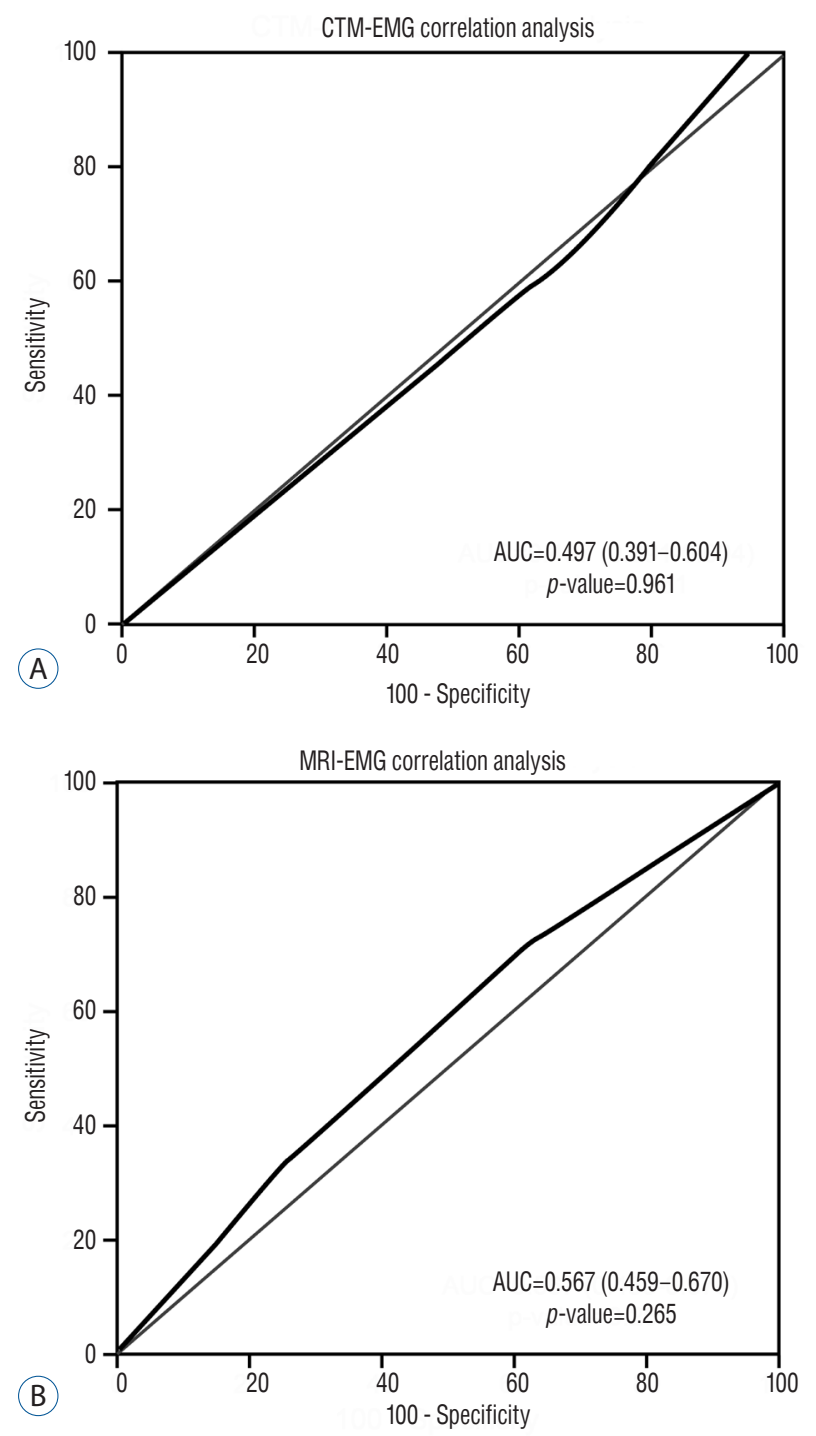

Fig. 4. A : Receiver-operating characteristic (ROC) curve for electromyography (EMG)-computed tomography-myelography (CTM) grading; the area under the curve $(A \cup C)$ is 0.497 , indicating no association between the EMG results and grades on CTM. B : ROC curve for EMG-magnetic resonance imaging (MRI) grading; the AUC is 0.567 , indicating no association between the EMG results and grades on MRI.

caused by nerve root compression due to a herniated disc, thickened ligamentum flavum, and hypertrophied articular facets in different locations along the route of the nerve root. In degenerative lumbar spine diseases, the mechanism of radicular leg pain may be explained in two ways: mechanical nerve root compromise and chemical irritation of the nerve $\operatorname{root}^{1)}$. Acute mechanical compression has been shown to result in root edema and inflammation ${ }^{1)}$. Therefore, nerve root compression grading on imaging studies is important for con-
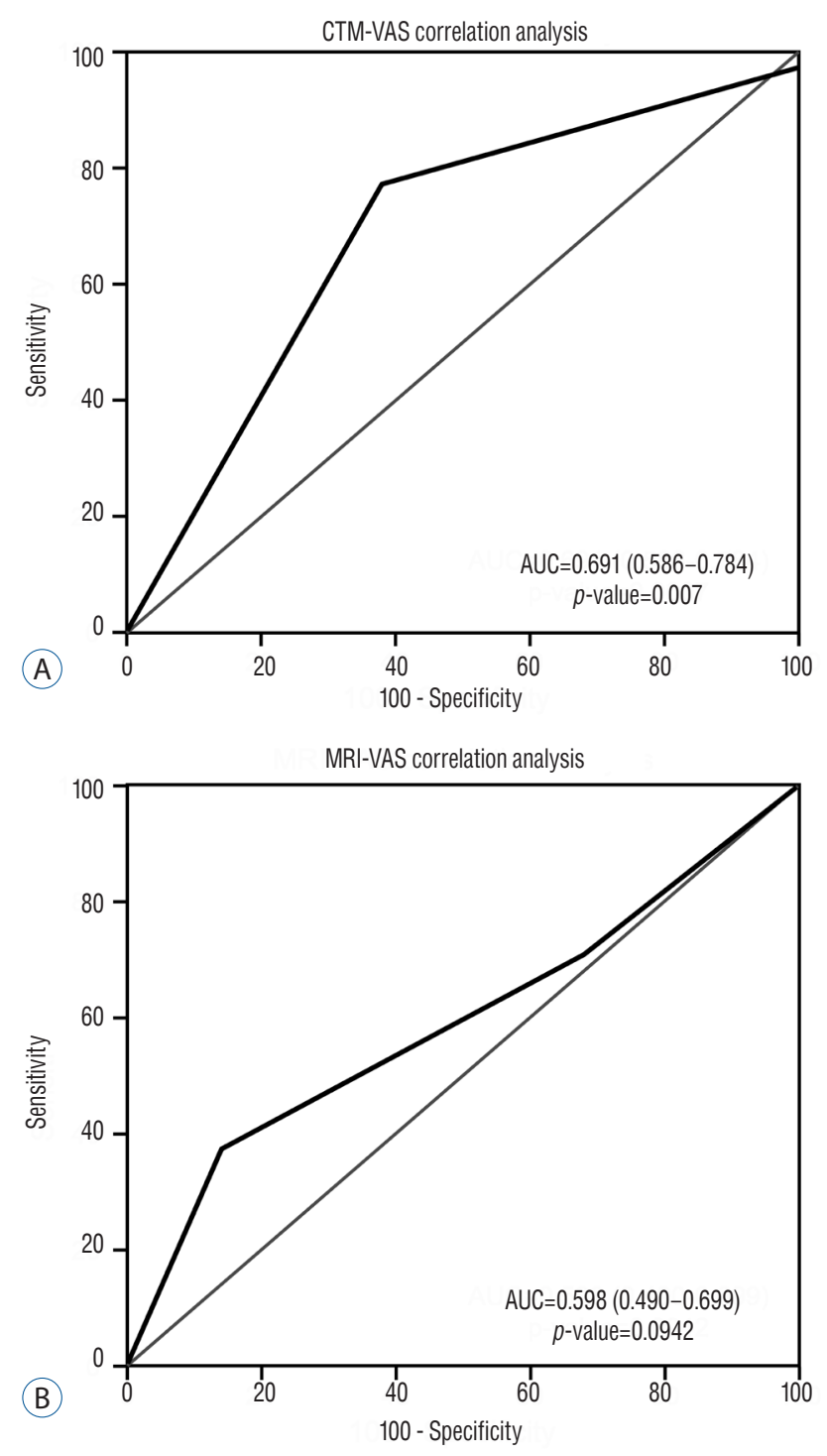

Fig. 5. A : Receiver-operating characteristic (ROC) curve for visual analog scale (VAS) score-computed tomography-myelography (CTM) grading; the area under the curve (AUC) was 0.691 , indicating an moderate association between VAS score and grade on CTM. B : The ROC curve for VAS score-magnetic resonance imaging (MRI) grading; the AUC is 0.598 , indicating less association between VAS score and grade on MRI.

firming radiculopathy.

In this study assessing the severity of nerve root compression both on MRI and CTM together, a proper grading system is a must; however, to the authors' knowledge, there has been no such a system so far ${ }^{1,9,20)}$. In the present study, the authors modified two previous grading systems applicable to both MRI and CTM in patients with radiculopathy caused by not only lumbar disc herniation but also lateral recess stenosis. 
Some studies comparing MRI and CTM using grade system of nerve root compression have been reported ${ }^{1,2,13,14,18,20)}$. Among these studies, the most representative are those by Pfirrmann et al. ${ }^{20)}$ and Bartynski and Lin ${ }^{1)}$. Pfirrmann et al. ${ }^{20)}$ prospectively investigated the correlation between nerve root compression grade on MRI and severity of nerve root compression in the surgical field only in patients with lumbar disc herniation. The study by Bartynski and $\operatorname{Lin}^{11}$ is unique in that nerve root compression grades on CTM and MRI were compared in patients with lateral recess abnormalities. In the study, the correlation between nerve root compression grades and clinical features using surgical nerve root compression grade was assessed retrospectively. In the study by Pfirrmann et al. ${ }^{20)}$, a grading system was used only for herniated lumbar discs, while in the study of Bartynski and Lin ${ }^{1)}$, a different grading system was used for lateral recess stenosis. Kang et al. ${ }^{16)}$ conducted a study that compared MRI, MR myelography, and myelography by using a grading system for nerve root compression. In the study, a modified grading system devised by Pfirrmann et al. ${ }^{20)}$ was used only for patients with lumbar disc herniation. The correlation between nerve root compression grades and clinical features assessed by using the VAS and Oswestry disability index was evaluated in the study ${ }^{16)}$.

Wildermuth et al. ${ }^{24)}$ reported a root compression grading classification for intervertebral foraminal stenosis on sagittal T1 MRI.

EMG is a useful electrophysiological test to confirm lumbar radiculopathy. Generally, given its low sensitivity, it is not considered an optimal screening test, but rather a confirmatory test $^{6,19)}$. However, to some extent, EMG can suggest the severity or extent of the disorder beyond the clinical symptoms by directly measuring the electrical changes of the compressed nerve $\operatorname{root}^{6,19)}$. However, in the present study, EMG findings did not correlate with the severity of nerve root compression on either CTM or MRI.

The VAS score is widely used in clinical research studies. It has been widely used to measure and monitor changes in functional outcomes in patients with radicular pain ${ }^{4,21)}$. Although the cause of radicular pain is not just nerve root compression but also chemical irritation, pain has been shown to be the single strongest predictor of the severity of nerve root compression in many cases of lumbar degenerative disease. In the present study, pain severity based on VAS score more highly correlated with changes in nerve root compression grades on CTM than with those on MRI.

These results show that CTM identifies clinical severity more accurately than MRI. Consequently, the above-mentioned results show that CTM has a stronger correlation with clinical features than MRI. Based on the above-mentioned results, CTM was more valuable than MRI in confirming symptomatic lumbar nerve root irritation caused by minimal nerve root compression and CTM grade was more congruent with the patients' clinical features.

Considering that the disc, ligament, bone, and nerve root each present with similar signals on T2-weighted axial MRI, the actual severity of the nerve root compression may be difficult to determine on axial MRI ${ }^{15)}$. However, bone, ligament, and nerve root are easier to differentiate on CTM axial scans, as these are accented by intrathecal contrast media ${ }^{10)}$. A contrast-filled rootlet appears to be more sensitive to minimal compression on CTM, and minimal root compression may be detected more easily on $\mathrm{CTM}^{2,10,13,14)}$. Among the 50 cases that showed different grading, 12 cases presented with MRI grade I nerve root compression that appeared as CTM grade III. Twenty-two patients were determined as having grade II nerve root compression on MRI, but grade III compression was observed on CTM. Illustrative cases are shown in Fig. 6.

According to the guideline by Swets ${ }^{11)}$, the accuracy of a diagnostic tool can be graded based on the AUC of the ROC curve (AUC $<0.5$ : noninformative, $0.5<$ AUC $<0.7$ : less accurate, $0.7<\mathrm{AUC}<0.9$ : moderately accurate, AUC $>0.9$ : highly accurate, $\mathrm{AUC}=1:$ perfect $)^{11)}$. Generally, the cutoff value is set at the point where the sum of the sensitivity and specificity is maximized in the ROC analysis and other clinical factors can be included ${ }^{11)}$. Other studies showed that the cutoff value, sensitivity, specificity, and AUC may change depending on the diagnostic criterion ${ }^{11,17)}$.

According to a study by Kang et al. ${ }^{17)}$ (obtaining a cutoff value for childhood obesity diagnosis using body fat percentage), a body mass index (BMI) of $\geq 85$ th percentile as a criterion for obesity had higher AUC and sensitivity (cutoff value : 34.5 of body fat percentage, sensitivity : 69.3 , and specificity : 74.2) than a BMI of $\geq 95$ th percentile as a criterion for obesity (cutoff value : 38.1 of body fat percentage, sensitivity : 50.5 , and specificity : 72.7). Thus, considering that a BMI of $\geq 85$ th percentile has higher AUC and sensitivity than a BMI of $\geq 95$ th percentile, a BMI of $\geq 85$ th percentile is a more appropriate diagnostic criterion for obesity in Kang et al.'s study ${ }^{17)}$. 
(A)
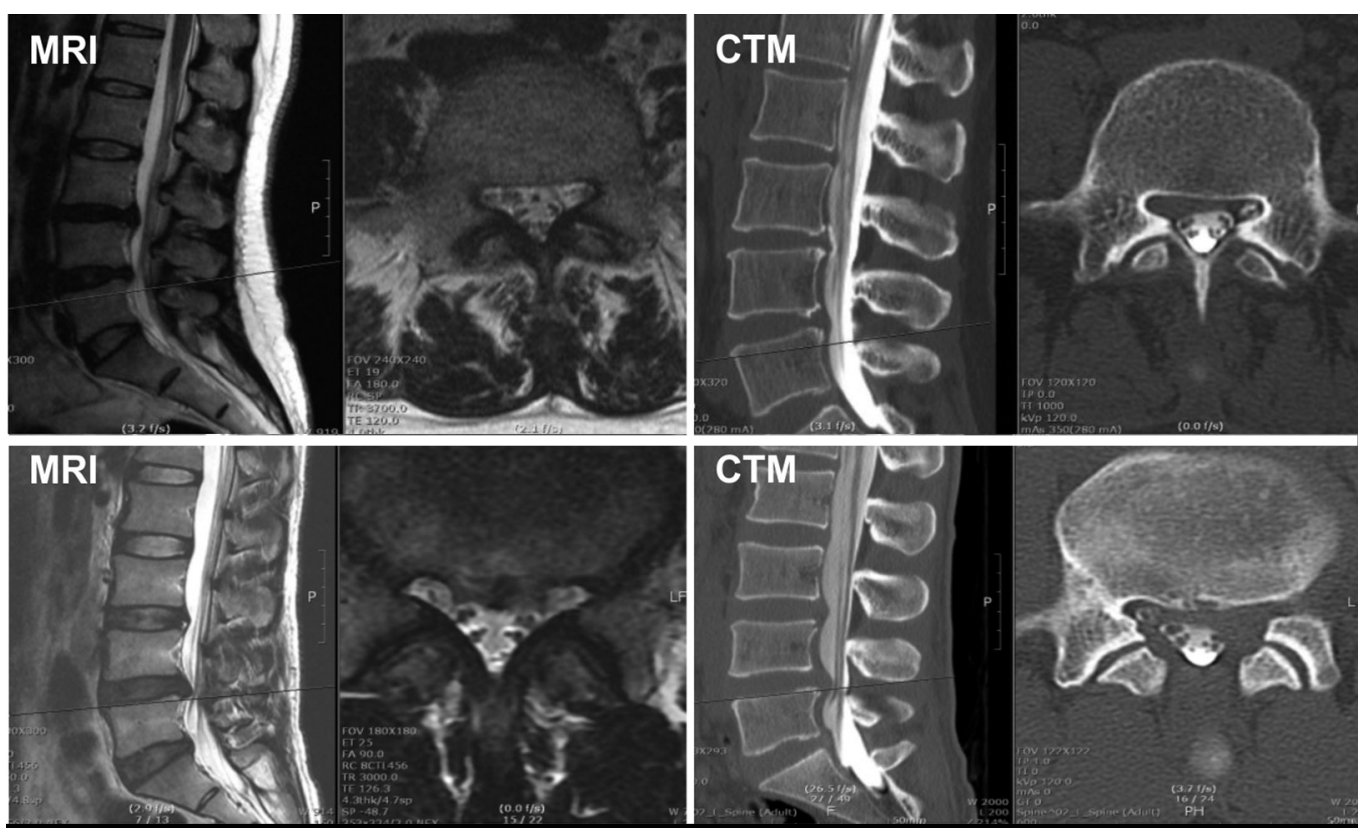

Fig. 6. A : Preoperative magnetic resonance imaging (MRI) T2 and computed tomography-myelography (CTM) axial scans showing differences in root compression finding. While the finding is grade I on the MRI axial scan, the finding is grade III on CTM. Grade I on MRI generally means less significant radicular pain that does not require surgical intervention. However, grade III on CTM generally means severe root compression that requires decompressive surgery. B : CTM axial scan showing severe left L5 root compression by obliteration of the nerve root not detectable on MRI axial scan. While grade II on MRI generally indicates a moderate degree of root compression, grade III on CTM generally means severe root compression that requires decompressive surgery. Grade III on CTM is congruent with the patients' clinical symptoms (severe radicular pain with a VAS score of 8).

In the same way, the cutoff value, sensitivity, specificity, and AUC can change according to the diagnostic criterion for radiculopathy based on VAS score division in this study. In this study, the authors set the VAS score of $\geq 6$ as a diagnostic criterion for radiculopathy to achieve the optimal diagnostic value (AUC 0.691, sensitivity 77.6, specificity 62.5).

Relatively little literature is available on the complications of myelography ${ }^{12,22)}$. Myelography is generally safe, with a low risk of infection; thus, limited literature is available that addresses the complication of myelography ${ }^{12,22)}$. The most significant complication of CTM was intracranial hypotension. Headache was observed in some cases but was improved after bed rest and hydration. None of the complications was severe, and all complications were temporary and improved spontaneously.

We analyzed the complication rate and details of complications by conducting a chart review. Complications were found in 17 (18\%) of 91 patients. Among the 17 patients with complications, 14 cases (82\%) presented with temporary mild back pain. All 14 cases were temporary mild back pain that occurred immediately after the myelography procedure.
None of the cases were clinically significant, and they were negligible on the detailed chart review. None of the patients had post-myelography infection, and $96 \%$ of the patients experienced no other clinically significant complications in the present study.

This study has several limitations. One limitation is the retrospective nature of the analysis. T1-weighted sagittal and axial images are useful for clarifying the relationship between the nerve root and epidural fat or thecal sac, and the use of these images might have affected the grading results. Comparisons between axial MRI and CTM scans obtained at slightly different levels were allowed in this study. Accepting the minuscule differences in the axial cutoff level of MRI and CTM could be a source bias.

Overly high mean intraclass correlation coefficient (ICC) values of the interobserver and intraobserver reliability $(>0.9)$ were likely possible because the same instructor from the same hospital trained the two neurosurgeons for 3 years and they shared their surgical indications with each other, which could also act as a source of bias. 


\section{CONCLUSION}

The present study demonstrates that CTM correlated more closely with clinical symptoms and tended to demonstrate more aggressive findings in the same patient than MRI. The results indicated that CTM might be more valuable than MRI in defining the severity of nerve root compression in patients with radiculopathy. In this regard, CTM can be a useful tool for confirmative diagnosis when MRI does not provide sufficient information to define an exact cause of radicular pain in patients with lumbar radiculopathy. Although most of the complications of CTM were mild and transient, with clinically insignificant symptoms, the invasiveness and potential complications of CTM, such as low intracranial pressure syndrome, should be investigated further.

\section{- Acknowledgements}

The preliminary results of this work were presented in International Society for the Advancement of Spine Surgery (ISASS 13) Conference, Vancouver (3-5 April 2013).

This study was approved by Institutional Review Board of Seoul St Mary's hospital; approval No. : KC12RASI0188. Due to the retrospective nature of the study, the need for informed consent was waived off.

\section{References}

1. Bartynski WS, Lin L : Lumbar root compression in the lateral recess: MR imaging, conventional myelography, and CT myelography comparison with surgical confirmation. AJNR Am J Neuroradiol 24 : 348-360, 2003

2. Bischoff RJ, Rodriquez RP, Gupta K, Righi A, Dalton JE, Whitecloud TS : A comparison of computed tomography-myelography, magnetic resonance imaging, and myelography in the diagnosis of herniated nucleus pulposus and spinal stenosis. J Spinal Disord 6 : 289-295, 1993

3. Botwin KP, Gruber RD : Lumbar spinal stenosis: anatomy and pathogenesis. Phys Med Rehabil Clin N Am 14 : 1-15, v, 2003

4. Breivik EK, Björnsson GA, Skovlund E : A comparison of pain rating scales by sampling from clinical trial data. Clin J Pain $16: 22-28,2000$

5. Burton CV, Kirkaldy-Willis WH, Yong-Hing K, Heithoff KB : Causes of failure of surgery on the lumbar spine. Clin Orthop Relat Res 157 : 191-199, 1981

6. Chiodo A, Haig AJ, Yamakawa KS, Quint D, Tong H, Choksi VR : Needle EMG has a lower false positive rate than MRI in asymptomatic older adults being evaluated for lumbar spinal stenosis. Clin Neurophysiol
$118: 751-756,2007$

7. Colosimo C, Gaudino S, Alexandre AM : Imaging in degenerative spine pathology. Acta Neurochir Suppl 108 : 9-15, 2011

8. Emch TM, Modic MT : Imaging of lumbar degenerative disk disease: history and current state. Skeletal Radiol 40 : 1175-1189, 2011

9. Fardon DF, Milette PC; Combined Task Forces of the North American Spine Society, American Society of Spine Radiology, and American Society of Neuroradiology : Nomenclature and classification of lumbar disc pathology. Recommendations of the combined task forces of the North American Spine Society, American Society of Spine Radiology, and American Society of Neuroradiology. Spine (Phila Pa 1976) 26 : E93E113, 2001

10. Grams AE, Gempt J, Förschler A : Comparison of spinal anatomy between 3-Tesla MRI and CT-myelography under healthy and pathological conditions. Surg Radiol Anat 32 : 581-585, 2010

11. Greiner $M$, Pfeiffer $D$, Smith RD : Principals and practical application of the receiver-operating characteristic analysis for diagnostic tests. Prev Vet Med $45:$ :23-41, 2000

12. Harreld JH, McMenamy JM, Toomay SM, Chason DP : Myelography: a primer. Curr Probl Diagn Radiol 40 : 149-157, 2011

13. Jackson RP, Cain JE Jr, Jacobs RR, Cooper BR, McManus GE : The neuroradiographic diagnosis of lumbar herniated nucleus pulposus: II. A comparison of computed tomography (CT), myelography, CT-myelography, and magnetic resonance imaging. Spine (Phila Pa 1976) 14 : 13621367, 1989

14. Janssen ME, Bertrand SL, Joe C, Levine MI : Lumbar herniated disk disease: comparison of MRI, myelography, and post-myelographic CT scan with surgical findings. Orthopedics 17 : 121-127, 1994

15. Jindal G, Pukenas $B$ : Normal spinal anatomy on magnetic resonance imaging. Magn Reson Imaging Clin N Am 19 : 475-488, 2011

16. Kang SH, Choi SH, Seong NJ, Ko JM, Cho ES, Ko KP : Comparative study of lumbar magnetic resonance imaging and myelography in young soldiers with herniated lumbar disc. J Korean Neurosurg Soc 48 : 501 505, 2010

17. Kang YS, Kang JH, Kim MC, Yu BY, Sung EJ, Lee SY, et al. : Cutoff of percent body fat to predict obesity and metabolic risk in children and adolescents: 2007 children and adolescent physical growth standard. Korean J Fam Med 30 : 887-894, 2009

18. Kent DL, Haynor DR, Larson EB, Deyo RA : Diagnosis of lumbar spinal stenosis in adults: a metaanalysis of the accuracy of $C T, M R$, and myelography. AJR Am J Roentgenol 158 : 1135-1144, 1992

19. Mondelli M, Aretini A, Arrigucci U, Ginanneschi F, Greco G, Sicurelli F : Clinical findings and electrodiagnostic testing in 108 consecutive cases of lumbosacral radiculopathy due to herniated disc. Neurophysiol Clin 43 : 205-215, 2013

20. Pfirrmann CW, Dora C, Schmid MR, Zanetti M, Hodler J, Boos N : MR image-based grading of lumbar nerve root compromise due to disk herniation: reliability study with surgical correlation. Radiology 230 : 583-588, 2004

21. Price $D D, M c G r a t h ~ P A$, Rafii $A$, Buckingham $B$ : The validation of visual analogue scales as ratio scale measures for chronic and experimental 
Diagnostic Value of Computed Tomography-Myelography I Park CK, et al.

pain. Pain $17:$ 45-56, 1983

22. Sandow BA, Donnal JF : Myelography complications and current practice patterns. AJR Am J Roentgenol 185 : 768-771, 2005

23. Song KJ, Choi BW, Kim GH, Kim JR : Clinical usefulness of CT-myelogram comparing with the MRI in degenerative cervical spinal disorders: is CTM still useful for primary diagnostic tool? J Spinal Disord Tech 22 :
353-357, 2009

24. Wildermuth S, Zanetti M, Duewell S, Schmid MR, Romanowski B, Benini $A$, et al. : Lumbar spine: quantitative and qualitative assessment of positional (upright flexion and extension) MR imaging and myelography. Radiology 207 : 391-398, 1998 